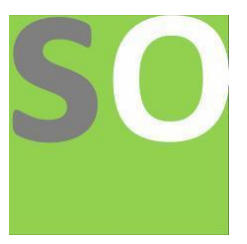

Article title: The possibility of the no-hair theorem being violated

Authors: Wen-Xiang Chen[1]

Affiliations: wxchen4277@qq.com[1]

Orcid ids: 0000-0002-0560-8280[1]

Contact e-mail: 954763891@qq.com

License information: This work has been published open access under Creative Commons Attribution License http://creativecommons.org/licenses/by/4.0/, which permits unrestricted use, distribution, and reproduction in any medium, provided the original work is properly cited. Conditions, terms of use and publishing policy can be found at https://www.scienceopen.com/.

Preprint statement: This article is a preprint and has not been peer-reviewed, under consideration and submitted to ScienceOpen Preprints for open peer review.

DOI: 10.14293/S2199-1006.1.SOR-.PPJRNRL.v1

Preprint first posted online: 30 March 2020

Keywords: No-hair theorem, numerical solution, superradiant stability 


\title{
The possibility of the no-hair theorem being violated
}

\author{
Wen-Xiang Chen* \\ Institute of quantum matter, \\ School of Physics and Telecommunication Engineering, \\ South China Normal University, \\ Guangzhou 510006, China
}

\begin{abstract}
Recently, one of my articles got some interesting results about Kerr black hole superradiant stability. That result contained some conclusions that violated the "No Hair Theorem". We know that the "No Hair Theorem" is the inference product of general relativity. I boldly believe that it may lead to a little progress in cosmological research.
\end{abstract}

Keywords: No-hair theorem, numerical solution, superradiant stability

\section{INTRODUCTION}

As stars evolve to the end, serious "collapse" will occur due to the inability to perform higher-level nuclear fusion to resist gravitational forces. The collapse result will be greatly different due to the gravitational gap caused by its mass. For example, the sun will eventually become a white dwarf Stars that are more than three times larger than the Sun will eventually become "black holes". This limit is called the Oppenheimer limit. Currently, accepted theory holds that there are only three physical quantities that a black hole can measure: mass, charge, and angular momentum. That is, for a black hole, once these three physical quantities are determined, the characteristics of the black hole are uniquely determined. This is called the black hole's no-hair theorem, or the uniqueness theorem of black holes. On the other hand, once a black hole is formed, other physical information before the formation of the black hole is lost. There are no cubes, cones or other convex forms on the black hole. This is another way to understand the black hole's no-hair theorem.

Regge and Wheeler [13] proved that the Schwarzschild black holes are stable under disturbance. Due to the superradiation phenomenon, the stability of rotating black holes becomes more complicated. The superradiation effect allows the extraction of rotational and Coulomb energy from rotating or charged black holes. In 1972, Press and Teukolsky[12] proposed that It is possible to add a mirror to the outside of a black hole to make a black hole bomb (according to the current explanation, this is a scattering process involving classical mechanics and quantum mechanics $[2,4,10,11])$.

When a bosonic wave is impinging upon a rotating black hole, the wave reflected by the event horizon will be amplified if the wave frequency $\omega$ lies in the following superradiant regime[12, 15, 16]

$$
0<\omega<m \Omega_{H}, \Omega_{H}=\frac{a}{r_{+}^{2}+a^{2}},
$$

where $m$ is azimuthal number of the bosonic wave mode, $\Omega_{H}$ is the angular velocity of black hole horizon. This

*Electronic address: wxchen4277@qq.com 
amplification is superradiant scattering. Therefore, through the superradiation process, the rotational energy or electromagnetic energy of a black hole can be extracted. If there is a mirror between the black hole's horizon and infinite space, the amplified wave will scatter back and forth and grow exponentially, which will cause the black hole's superradiant instability.

The no-hair theorem states that all black hole solutions of Einstein-Maxwell's gravitational and electromagnetic equations of general relativity can only be fully characterized by three classical parameters that can be observed from the outside: mass, charge, and angular momentum. All other information (metaphors about the "hair" that forms or falls into a black hole) "disappears" after the event horizon of the black hole, so external observers can never enter.

The no-hair theorem shows that for the disturbance incident on Kerr black hole, the geometry shown by its coupling with the black hole can be expressed by the parameters of the black hole.

From [3], $\mu=y \omega$, we get inequality (2)

$$
\frac{m a}{\frac{2 r_{-}^{2}}{y}+\left(M r_{-}-r_{-}^{2}\right) y+\left(y+\frac{1}{y}\right) \frac{a^{2}}{2 M}\left(M-r_{-}\right)}<\mu<\sqrt{2} m \Omega_{H},
$$

where $\mu$ is the mass.

That result contained some conclusions that violated the "No Hair Theorem" (This result shows that not all black hole solutions are only related to three black hole parameters).

\section{DESCRIPTION OF THE KERR-BLACK-HOLE SYSTEM}

The metric of the Kerr black hole[6, 8, 9] (in natural unit $\mathrm{G}=\mathrm{c}=1$ ) is

$$
\begin{gathered}
d s^{2}=\frac{\Delta}{\rho^{2}}\left(d t-a \sin ^{2} \theta d \phi\right)^{2}+\frac{\rho^{2}}{\Delta} d r^{2}+\rho^{2} d \theta^{2}+\frac{\sin ^{2} \theta}{\rho^{2}}\left[a d t-\left(r^{2}+a^{2}\right) d \phi\right]^{2} . \\
\Delta=r^{2}-2 M r+a^{2}, \rho^{2}=r^{2}+a^{2} \cos ^{2} \theta .
\end{gathered}
$$

Regarding the scalar field, we limit it with the Klein-Gordon equation

$$
\left(\nabla^{\nu} \nabla_{\nu}-\mu^{2}\right) \Psi=0
$$

Eigenvalues of the above formula and spherical harmonic functions(The integer parameters $m$ and $l \geq|m|$ are the azimuthal and spheroidal harmonic indices of the scalar field modes) can be written as

$$
\Psi_{\operatorname{lm}}(\mathrm{t}, \mathrm{r}, \theta, \phi)=\sum_{\mathrm{l}, \mathrm{m}} \mathrm{e}^{\mathrm{im} \phi} \mathrm{S}_{\operatorname{lm}}(\theta) \mathrm{R}_{\operatorname{lm}}(\mathrm{r}) \mathrm{e}^{-\mathrm{i} \omega \mathrm{t}}
$$

Substituting (6) into the Klein-Gordon wave equation, we can find that the angular function $\mathrm{S}_{\operatorname{lm}}(\theta)$ satisfies the angular motion equation $[1,5,14]$

$$
\frac{1}{\sin \theta} \frac{d\left(\sin \theta \frac{d S_{l m}}{d \theta}\right)}{d \theta}+\left[K_{l m}+a^{2}\left(\mu^{2}-\omega^{2}\right) \sin ^{2} \theta-\frac{m^{2}}{\sin ^{2} \theta}\right] S_{l m}=0 ;
$$

According to the references[1, 5, 14],we get inequality $(8)$

$$
K_{l m} \geq m^{2}-a^{2}\left(\mu^{2}-\omega^{2}\right)
$$

where $l$ is the spherical harmonic index, $m$ is the azimuthal harmonic index with $-l \leq m \leq l$ and $\omega$ is the energy of the mode. 


\section{KERR-BLACK-HOLE-MASSIVE-SCALAR-FIELD SYSTEM AND SUPERRADIANT INSTABILITY REGIME}

The radial Klein-Gordon equation $[5,14]$ obeyed by $R_{l m}$ is given by

$$
\Delta \frac{d\left(\Delta \frac{d R}{d r}\right)}{d r}+U R=0
$$

where

$$
\Delta=r^{2}-2 M r+a^{2}
$$

and

$$
U=\left[\omega\left(\mathrm{r}^{2}+a^{2}\right)-\mathrm{m} a\right]^{2}+\Delta\left[2 \mathrm{~m} a \omega-\mu^{2}\left(\mathrm{r}^{2}+a^{2}\right)-K_{l m}\right]
$$

The inner and outer horizons of the black hole are

$$
r_{ \pm}=M \pm \sqrt{M^{2}-a^{2}}
$$

and we get that

$$
r_{+}+r_{-}=2 M, r_{+} r_{-}=a^{2}
$$

The radial potential equation is transformed into a tortoise coordinate wave equation to solve the asymptotic behavior of the wave function.We use tortoise coordinate $r_{*}$ by equation $\frac{d r_{*}^{2}}{d r^{2}}=\frac{r^{2}}{\Delta}$ and another radial function $\psi=\mathrm{rR}$.We get the following radial wave equation

$$
\frac{\mathrm{d}^{2} \psi}{\mathrm{dr}_{*}^{2}}+V \psi=0
$$

where

$$
V=\frac{U}{r^{4}}-\frac{2 \Delta}{r^{6}}\left(M r-a^{2}\right)
$$

Then we get the asymptotic solutions for the radial wave equation below

$$
\begin{gathered}
r \rightarrow \infty\left(\mathrm{r}_{*} \rightarrow \infty\right) \Rightarrow R_{\operatorname{lm}} \sim \frac{1}{r} e^{-\sqrt{\mu^{2}-\omega^{2}} r_{*}}, \\
r \rightarrow r_{+}\left(r_{*} \rightarrow-\infty\right) \Rightarrow R_{\operatorname{lm}} \sim e^{-i\left(\omega-m \Omega_{H}\right) r_{*}} .
\end{gathered}
$$

When

$$
\omega^{2}-\mu^{2}<0
$$

there is a bound state of the scalar field.

When $\varphi=\Delta^{\frac{1}{2}} R$, radial potential equation(9) can be transformed into the flat space-time wave equation

$$
\frac{d^{2} \varphi}{d r^{2}}+\left(\omega^{2}-V_{1}\right) \varphi=0, V_{1}=\omega^{2}-\frac{U+M^{2}-a^{2}}{\Delta^{2}}
$$


It was previously proved[8]that, for a scalar field of proper mass $\mu$ interacting with a Kerr black hole of angular velocity $\Omega_{H}$, the inequality

$$
\mu<\sqrt{2} \mathrm{~m} \Omega_{H}
$$

fixed Kerr black hole-mass-scale scalar field configuration upper bound.

Although much research has been done on the superradiance of rotating black holes, even Kerr black holes have not been thoroughly studied.Hod proved[8] that one finds the upper bound

$$
\mu<\mathcal{F}(\gamma) \cdot m \Omega_{\mathrm{H}}
$$

on the scalar mass of fixed bound state field configuration, where $\gamma=\frac{r_{-}}{r_{+}}$, and the dimensionless function $\mathcal{F}=\mathcal{F}(\gamma)$ is given by

$$
\mathcal{F}(\gamma)=\sqrt{\frac{2(1+\gamma)\left(1-\sqrt{1-\gamma^{2}}\right)-\gamma^{2}}{\gamma^{3}}} .
$$

In Table 1 we present the dimensionless ratio $\mu_{\text {numerical }} / \mu_{\text {bound }}$, where $\mu_{\text {numerical }}$ is the numerically computed field masses which mark the onset of the superradiant instabilities in the composed Kerr-black-hole-massive-scalar-field system, and $\mu_{\text {bound }}$ is the analytically derived upper bound(21) on the superradiant instability regime of the composed black-hole-field system. One finds from Table 1 that the superradiant instability regime of the composed Kerr-blackhole-massive-scalar-field system is characterized by the relation $\mu_{\text {numerical }} / \mu_{\text {bound }}<1$.Table 1 :

\begin{tabular}{|c|c|c|c|}
\hline$s \equiv a / M$ & $\mathcal{F}(s)$ & $\frac{\mu(l=m=1)}{\mu_{\text {bound }}}$ & $\frac{\mu(l=m=10)}{\mu_{\text {bound }}}$ \\
\hline 0.1 & 1.00031 & 0.99977 & 0.99940 \\
0.2 & 1.00129 & 0.99903 & 0.99967 \\
0.3 & 1.00301 & 0.99774 & 0.99948 \\
0.4 & 1.00567 & 0.99573 & 0.99901 \\
0.5 & 1.00960 & 0.99276 & 0.99776 \\
0.6 & 1.01541 & 0.98835 & 0.99715 \\
0.7 & 1.02437 & 0.98163 & 0.99558 \\
0.8 & 1.03955 & 0.96995 & 0.99276 \\
0.9 & 1.07168 & 0.94694 & 0.98676 \\
0.95 & 1.11039 & 0.91878 & 0.97942 \\
0.99 & 1.21646 & 0.84910 & 0.96165 \\
0.999 & 1.37370 & 0.76084 & 0.94280 \\
\hline
\end{tabular}

Hod proved[7] that the Kerr black hole should be superradiantly stable under massive scalar perturbation when $\mu \geq \sqrt{2} m \Omega_{H}$, where $\mu$ is the mass. $\mathcal{F}(s)$ and $\mathcal{F}(\gamma)$ are functions of the parameters of the black hole.We know that the numerical solutions to the superradiant instability must be outside the intersection of of $\mu \geq \sqrt{2} m \Omega_{H}$ and $0<\omega<m \Omega_{H}$. We see that the numerical solutions of the Kerr black hole's superradiant instability are consistent with the no-hair theorem(For the effective potential of the Kerr black hole has a potential well outside the horizon, superradiant instability occurs at that time. And the numerical solutions for the superradiant instability are obtained by combining the above conditions with the no-hair theorem)[6,8].From Table 1 , we know that some numerical solutions to the superradiant instability must be in the intersection of $\mu<\sqrt{2} \mathrm{~m} \Omega_{H}$ and $0<\omega<m \Omega_{H}$. 


\section{THAT RESULT CONTAINED SOME CONCLUSIONS THAT VIOLATED THE "NO HAIR THEOREM'}

For $\mu^{2}=y^{2} \omega^{2}$, when $y>4.352$, there exists a certain interval to let the inequality

$$
\frac{m a}{\frac{2 r_{-}^{2}}{y}+\left(M r_{-}-r_{-}^{2}\right) y+\left(y+\frac{1}{y}\right) \frac{a^{2}}{2 M}\left(M-r_{-}\right)}<\mu<\sqrt{2} m \Omega_{H},
$$

be satisfied. So the Kerr black hole is superradiantly stable at that time [3].

Because $y$ can theoretically be taken in the range of $y>4.352$, we see that when $y$ is relatively large(according to the properties of the boson, $y$ can be very large), the superradiant stability interval of $\mu$ can always include some numerical solutions of the superradiant instability obtained by previous people. If I'm right, there might be something wrong with the no-hair theorem. This result shows that not all black hole solutions are only related to three black hole parameters.In this case, it also depends on the value of $y$.

We do a thinking experiment. When the incident disturbance parameter $y$ satisfies the above conditions $(y$ is very large, so $\mu$ can always include the numerical solutions of the superradiant instability ), if it can produce instability, then the no-hair theorem is still correct. If we observe that the Kerr black hole is stable, then the no-hair theorem is problematic.

\section{SUMMARY}

Recently, an article of mine has obtained interesting results about the superradiant stability of Kerr black holes. The result contains some conclusions that violate the "no-hair theorem".I boldly believe that it may lead to a little progress in cosmological research.

[1] Berti, E., Cardoso, V. and Casals, M. [2006], 'Eigenvalues and eigenfunctions of spin-weighted spheroidal harmonics in four and higher dimensions', Physical Review D 73(2), 024013.

[2] Cardoso, V., Dias, O. J., Lemos, J. P. and Yoshida, S. [2004], 'Black-hole bomb and superradiant instabilities', Physical Review D 70(4), 044039.

[3] Chen, W.-X. and Huang, Z.-Y. [2019], 'Superradiant stability of the kerr black hole', International Journal of Modern Physics D.

URL: https://doi.org/10.1142/S0218271820500091

[4] Greiner, W., Müller, B. and Rafelski, J. [1985], Evolution of the vacuum state in supercritical potentials, in 'Quantum Electrodynamics of Strong Fields', Springer, pp. 257-299.

[5] Hartman, T., Song, W. and Strominger, A. [2010], 'Holographic derivation of kerr-newman scattering amplitudes for general charge and spin', Journal of High Energy Physics 2010(3), 118.

[6] Herdeiro, C. A. and Radu, E. [2014], 'Kerr black holes with scalar hair', Physical review letters 112(22), 221101.

[7] Hod, S. [2012], 'On the instability regime of the rotating kerr spacetime to massive scalar perturbations', Physics Letters B 708(3-5), 320-323.

[8] Hod, S. [2016], 'The superradiant instability regime of the spinning kerr black hole', Physics Letters B 758, 181-185. 
[9] Kerr, R. P. [1963], 'Gravitational field of a spinning mass as an example of algebraically special metrics', Physical review letters 11(5), 237.

[10] Manogue, C. A. [1988], 'The klein paradox and superradiance', Annals of Physics 181(2), 261-283.

[11] Penrose, R. [2002], 'Gravitational collapse: The role of general relativity (reprinted from rivista del nuovo cimento, numero speziale, i, vol 257, 1969)', General Relativity and Gravitation 34(7), 1141-1165.

[12] Press, W. H. and Teukolsky, S. A. [1973], 'Perturbations of a rotating black hole. ii. dynamical stability of the kerr metric', The Astrophysical Journal 185, 649-674.

[13] Regge, T. and Wheeler, J. A. [1957], 'Stability of a schwarzschild singularity', Physical Review 108(4), 1063.

[14] Teukolsky, S. A. [1973], 'Perturbations of a rotating black hole. 1. fundamental equations for gravitational electromagnetic and neutrino field perturbations', Astrophys. J. 185, 635-647.

[15] Vilenkin, A. [1978], 'Exponential amplification of waves in the gravitational field of ultrarelativistic rotating body', Physics Letters B 78(2-3), 301-303.

[16] Zel'dovich, Y. B. [1972], 'Pis' ma zh. eksp. teor. fiz. 14, 270 (1971)[jetp lett. 14, 180 (1971)]', Zh. Eksp. Teor. Fiz 62 , 2076. 